\title{
Weed Management in Late Kharif Cluster Bean (Cyamopsis tetragonoloba (L.) Taub) and its Impact on Crop Growth and Yield
}

\author{
Piyush Kumar Saras*, B. D. Patel, Sejal K. Parmar and R. B. Patel
}

DWSR-Anand Centre, B. A. College of Agriculture, Anand Agricultural University, Anand, Gujarat (388 110), India

\section{Article History}

Manuscript No. AR1302

Received in $16^{\text {th }}$ February, 2015

Received in revised form $18^{\text {th }}$ January, 2016

Accepted in final form $7^{\text {th }}$ February, 2016

\section{Correspondence to}

*E-mail: saras.piyush11@gmail.com

\section{Keywords}

Herbicides, cluster bean, weed control efficiency, yield

\begin{abstract}
A field experiment was conducted during kharif 2013-2014 at DWSR-Anand Centre, Anand Agricultural University, Anand (Gujarat) where four herbicides either as sole, integrated with hand weeding (HW) or sequentially applied were evaluated for efficacies of the herbicides on controlling weeds, their influences on yield and production economics on cluster bean (Cyamopsis tetragonoloba (L.) Taub) variety was Gujarat Gaur 1 (GG-1). The dominant weed species among monocot weeds were Echinochola crusgalli, Eleusine indica, Commelina benghalensis, Digitaria sanguinalis, Eragrostis major, Cynodon dactylon and Cyperus rotundus, however among dicot weed species Digera arvensis, Phyllanthus niruri, Amaranthus viridis, Oldenlandia umbellate, Euphorbia hirta and Spergula arvensis were observed during the growing season. Weed dry weight of monocot and dicot weed was the lowest in intercultering followed by hand weeding carried out at 20 and 40 days after sowing at 25 and 50 days after sowing and at harvest which remain at par with integrated weed management approach treatment pendimethalin+imazethapyr (pre-mixed) @ $800 \mathrm{~g}$ ha $^{-1}$ pre emergence followed by hand weeding at 30 days after sowing. Maximum weed control efficiency was observed with the intercultering followed by hand weeding at 20 and 40 days after sowing. This treatment also recorded higher yield attributes and seed and stover yield (16.55 and $\left.49.36 \mathrm{q} \mathrm{ha}^{-1}\right)$. And gave maximum net monetary returns and $\mathrm{B}: \mathrm{C}$ ratio ( $₹ 59404 \mathrm{ha}^{-1}$ and 2.97 , respectively).
\end{abstract}

\section{Introduction}

Cluster bean is one of the important drought resistant, hardy, deep-rooted short-duration and highly adapted to the harsh edapho-climatic conditions of hot arid zone of India. In recent year, besides, its conventional uses human consumption and cattle feed. It has emerged out as an industrial crop, due to presence of galactomannan (30-35\%) in its endosperm. Its gum is used in industries such as in food processing, paints, cosmetics, pharmaceuticals, synthetic resins, water blocking agents in explosives and as a thickener, fire retardant etc (Gandhi et al., 2005). Besides it, cluster bean gum is extensively used as a sizing agent in paper and textile industries as an effective flocculent and filterant in mining and metallurgical processes (Jain et al., 1987). For medicinal uses, the plants are burnt to ash, mixed with oil and used as poultice on cattle boils. Green leaves are eaten to cure night-blindness. Seeds are used as a chemotherapecitic agent against small pox. Boiled cluster bean seeds are used as a poultice on enlarged liver; head swelling and on swellings due to broken bones. Seeds are also used as a laxative (Arora, 1979).

It is cultivated in about $2.96 \mathrm{~m}$ ha with annual production of $10.59 \mathrm{mt}$ with $358.0 \mathrm{~kg} \mathrm{ha}^{-1}$ of average productivity in India (Anonymous, 2012). Rajasthan contributes $1.82 \mathrm{~m}$ ha with production $1.99 \mathrm{mt}$ (Anonymous, 2011). Amongst agronomic factors known to augment crop production appropriate weed management is considered to be important. Poor weed management is one of the important factor for low yield of this crop. Slow growth at initial stages of the crop favors recurrent flushes of weeds, which compete with crop for essentials of growth and cause heavy reduction in its seed yield. Critical period for crop-weed competition in cluster bean has been identified as 20 to 30 days after sowing and presence of weeds showed in yield reductions by 47 to $92 \%$ (Punia et al., 2011). Among different weed management practices, use of herbicides is the only choice under adverse situations. However, herbicides are costly and their availability in desired quality has residual effect. Therefore, integrated approach (chemical and mechanical control) makes weed management 
more effective and economical.

\section{Materials and Methods}

The experiment was conducted during the kharif season of the year 2013-14 at the farm of the DWSR-Anand Centre, B.A. College of Agriculture, Anand Agricultural University, Anand (Gujarat). The soil of the experimental site was loamy sand having $7.8 \mathrm{pH}, 0.27 \%$ Organic Carbon and $342 \mathrm{~kg}$ Nitrogen $\mathrm{ha}^{-1}, 48 \mathrm{~kg} \mathrm{P}_{2} \mathrm{O}_{5} \mathrm{ha}^{-1}$ and $298 \mathrm{~kg} \mathrm{~K}_{2} \mathrm{O} \mathrm{ha}{ }^{-1}$. The experiment was carried out in randomized block design with four replications. The experiment consist of ten treatments viz.,pendimethalin@750 $\mathrm{g} \mathrm{ha}^{-1}$ pre-emergence, pendimethalin@750 g ha ${ }^{-1}$ pre-emergence followed by hand weeding at 30 days after sowing, imazethapyr (a) $75 \mathrm{~g} \mathrm{ha}^{-1}$ pre-emergence, imazethapyr @ $75 \mathrm{~g} \mathrm{ha}^{-1}$ preemergence followed byhand weeding at 30 days after sowing, propaquizafop@75 g ha h $^{-1}$ post emergence,propaquizafop@ $75 \mathrm{~g} \mathrm{ha}^{-1}$ post emergence followed by hand weeding at 30 days after sowing, pendimethalin+imazethapyr (pre-mixed) @ 800 $\mathrm{g} \mathrm{ha}^{-1}$ pre-emergence, pendimethalin+imazethapyr (pre-mixed) (a) $800 \mathrm{~g} \mathrm{ha}^{-1}$ pre-emergence followed by hand weeding at 30 days after sowing, interculturing followed by hand weeding at 20 and 40 days after sowing and compared with weedy check. Recommended dose of fertilizers Nitrogen $\left(20 \mathrm{~kg} \mathrm{ha}^{-1}\right)$ and $\mathrm{P}_{2} \mathrm{O}_{5}$ (40 $\mathrm{kg} \mathrm{ha}^{-1}$ ) were applied through urea and SSP, respectively. The herbicides were applied with their respective doses as per treatments. Spraying was done with flat fen nozzle with knapsack sprayer using 500 liter $\mathrm{ha}^{-1}$. Weed population and dry weight of weeds were taken using quadrate of $0.25 \mathrm{~m}^{2}$ size at 30 and 60 days after sowing and at harvest. Weed data were subjected to square root transformation before statistical analysis. Growth and yield attribute characters, seed and stover yield recorded and economics were also calculated.

\section{Results and Discussion}

\subsection{Effects on weeds}

The experimental field was dominated by Echinochola crus galli, Eleusine indica, Commelina benghalensis, Digitaria sanguinalis, Eragrostis major, Cynodon dactylon and Cyperus rotundus of monocot weeds and Digera arvensis, Phyllanthus niruri, Amaranthus viridis, Oldenlandia umbellate, Euphorbia hirta and Spergula arvensis of dicot weeds during the growing season, similar results were reported by Yadav et al. (2011). The weed density and weed dry weight was significantly differ with the weed control practices (Table 1). Among the different weed management practices, interculturing followed by hand weeding at 20 and 40 days after sowing recorded significantly lower density of monocot and dicot weedsand weed dry weight at 25 and 50 days after sowing than rest of the treatments but it was remained at pat with pendimethalin+imazethapyr (pre-mixed)@800 g ha h $^{-1}$ pre-emergence followed by hand weeding at 30 days after sowing at 50 days after sowing and at harvest. At all the stages, interculturing followed by hand weeding at 20 and 40 days after sowing $\left(\mathrm{T}_{9}\right)$ gave the best management of monocot and dicot weeds than other treatments because initially weeds were controlled by interculturing and hand weeding carried out at 20 days after sowing and whatever weeds emerged later were effectively removed by subsequent interculturing and hand weeding carried out at 40 days after sowing. Effective control of weeds through cultural practices was also reported by Yadav et al. (2011). In the long run, pre-mixture of pendimethalin+imazethapyr @ $800 \mathrm{~g}$ $\mathrm{ha}^{-1}$ pre-emergence was found more effective for controlling monocot weeds as well as dicot weeds. This might be due to the broad spectrum control of weeds because of combination of two herbicides with different mode of action and decreasing residual effect of pendimethalin and prolonged residual effect of imazethapyr. The result conformity was found with Soltani et al. (2012); Jha and Soni (2013). The weed density and dry weight of monocot and dicot weeds in control plot were significantly the highest than rest of the treatments.

\subsection{Weed index and weed control efficiency}

Minimum weed index (1.94\%) and maximum weed control efficiency (Table 1) at 25 and 50 days after sowing and at harvest were observed with interculturing followed by hand weeding carried out at 20 and 40 days after sowing. The lower weed index and higher weed control efficiency with interculturing followed by hand weeding at 20 and 40 days after sowing, higher efficacy of the herbicides at early growth stage and one hand weeding at later stage was effective in controlling weed dry matter in the different integrated approaches of weed management. The well-developed foliage canopy, intercepting solar energy effectively covered the ground area which reduced the weed emergence and increased the weed control efficacy. May be contributed to the lowest weed competition and resulted higher seed yield (Table 2). Yadav et al. (2011) also reported higher weed control efficiency in hand weeding at 20 and 40 days after sowingin cluster bean crop.

\subsection{Yield attributes and yield}

The higher plant height of cluster bean was recorded at 60 days after sowing andat harvest under interculturing followed by hand weeding at 20 and 40 days after sowingand at 30 days after sowing higher in weedy check due to weed competition for light (Table 2). Crop dry matter accumulation at 50 days after sowing, number of branches, number of pods plant ${ }^{-1}$ and test weight were recorded significantly higher at harvest under 
Table 1: Weed density, weed dry weight and weed control efficiency at different days influenced by different weed management practices

\begin{tabular}{|c|c|c|c|c|c|c|c|c|c|c|c|c|c|}
\hline \multirow{3}{*}{$\begin{array}{l}\text { Treat- } \\
\text { ments }\end{array}$} & \multicolumn{4}{|c|}{ Density of weeds (no. $\mathrm{m}^{-2}$ ) } & \multicolumn{6}{|c|}{ Dry weight of weeds (g) } & \multirow{2}{*}{\multicolumn{3}{|c|}{$\begin{array}{c}\text { Weed control efficiency } \\
(\%)\end{array}$}} \\
\hline & \multicolumn{2}{|c|}{ Monocot } & \multicolumn{2}{|c|}{ Dicot } & \multicolumn{3}{|c|}{ Monocot } & \multicolumn{3}{|c|}{ Dicot } & & & \\
\hline & $\begin{array}{c}25 \\
\text { DAS }\end{array}$ & $\begin{array}{c}50 \\
\text { DAS }\end{array}$ & $\begin{array}{c}25 \\
\text { DAS }\end{array}$ & $\begin{array}{c}50 \\
\text { DAS }\end{array}$ & $\begin{array}{c}25 \\
\text { DAS }\end{array}$ & $\begin{array}{c}50 \\
\text { DAS }\end{array}$ & $\begin{array}{c}\text { At } \\
\text { harvest }\end{array}$ & $\begin{array}{c}25 \\
\text { DAS }\end{array}$ & $\begin{array}{c}50 \\
\text { DAS }\end{array}$ & $\begin{array}{c}\text { At har- } \\
\text { vest }\end{array}$ & $\begin{array}{c}25 \\
\text { DAS }\end{array}$ & $\begin{array}{c}50 \\
\text { DAS }\end{array}$ & $\begin{array}{c}\text { At } \\
\text { Harvest }\end{array}$ \\
\hline $\mathrm{T}_{1}$ & $\begin{array}{c}7.55^{\mathrm{d}} \\
(56.75)\end{array}$ & $\begin{array}{c}6.05^{\mathrm{ab}} \\
(36.50)\end{array}$ & $\begin{array}{c}7.29^{\mathrm{d}} \\
(53.0)\end{array}$ & $\begin{array}{l}6.35^{\mathrm{bc}} \\
(40.0)\end{array}$ & $\begin{array}{c}5.26^{\mathrm{d}} \\
(27.24)\end{array}$ & $\begin{array}{l}{ }^{2.97^{b}} \\
(8.40)\end{array}$ & $\begin{array}{c}2.92^{\mathrm{c}} \\
(8.25)\end{array}$ & $\begin{array}{l}4.53^{\mathrm{d}} \\
(20.1)\end{array}$ & $\begin{array}{l}2.54^{\mathrm{bc}} \\
(6.00)\end{array}$ & $\begin{array}{c}6.98^{\mathrm{bc}} \\
(48.50)\end{array}$ & 55.38 & 35.16 & 43.56 \\
\hline $\mathrm{T}_{2}$ & $\begin{array}{c}8.10^{\mathrm{cd}} \\
(65.25)\end{array}$ & $\begin{array}{c}5.09^{\mathrm{bc}} \\
(25.75)\end{array}$ & $\begin{array}{c}7.05^{\mathrm{d}} \\
(49.2)\end{array}$ & $\begin{array}{c}5.25^{\mathrm{d}} \\
(27.25)\end{array}$ & $\begin{array}{c}5.63^{\mathrm{cd}} \\
(31.32)\end{array}$ & $\begin{array}{l}2.57^{\mathrm{bc}} \\
(6.21)\end{array}$ & $\begin{array}{l}0.71^{\mathrm{d}} \\
(0.00)\end{array}$ & $\begin{array}{l}4.38^{\mathrm{d}} \\
(18.7)\end{array}$ & $\begin{array}{l}2.14^{\mathrm{d}} \\
(4.09)\end{array}$ & $\begin{array}{c}6.08^{\mathrm{d}} \\
(36.75)\end{array}$ & 53.24 & 53.31 & 64.29 \\
\hline $\mathrm{T}_{3}$ & $\begin{array}{c}3.89^{\mathrm{e}} \\
(15.50)\end{array}$ & $\begin{array}{c}5.31^{\mathrm{bc}} \\
(28.00)\end{array}$ & $\begin{array}{l}2.28^{\mathrm{e}} \\
(4.75)\end{array}$ & $\begin{array}{c}5.44^{\mathrm{d}} \\
(29.25)\end{array}$ & $\begin{array}{c}2.74^{\mathrm{e}} \\
(7.44)\end{array}$ & $\begin{array}{l}2.62^{\mathrm{bc}} \\
(6.44)\end{array}$ & $\begin{array}{c}4.88^{\mathrm{b}} \\
(23.50)\end{array}$ & $\begin{array}{c}1.51^{\mathrm{e}} \\
(1.81)\end{array}$ & $\begin{array}{l}2.21^{\mathrm{d}} \\
(4.39)\end{array}$ & $\begin{array}{c}3.76^{\mathrm{e}} \\
(13.75)\end{array}$ & 91.42 & 51.03 & 63.53 \\
\hline $\mathrm{T}_{4}$ & $\begin{array}{c}3.77^{\mathrm{e}} \\
(14.00)\end{array}$ & $\begin{array}{c}4.69^{\mathrm{c}} \\
(22.00)\end{array}$ & $\begin{array}{c}2.23^{\mathrm{e}} \\
(4.50)\end{array}$ & $\begin{array}{c}5.15^{\mathrm{de}} \\
(26.25)\end{array}$ & $\begin{array}{l}2.67^{\mathrm{e}} \\
(6.72)\end{array}$ & $\begin{array}{c}2.34^{\mathrm{c}} \\
(5.06)\end{array}$ & $\begin{array}{c}5.26^{\mathrm{ab}} \\
(27.50)\end{array}$ & $\begin{array}{c}1.49^{\mathrm{e}} \\
(1.71)\end{array}$ & $\begin{array}{l}2.10^{\mathrm{de}} \\
(3.94)\end{array}$ & $\begin{array}{c}2.67^{\mathrm{f}} \\
(6.75)\end{array}$ & 92.15 & 59.83 & 66.38 \\
\hline $\mathrm{T}_{5}$ & $\begin{array}{c}9.55^{\mathrm{b}} \\
(91.25)\end{array}$ & $\begin{array}{c}5.17^{\mathrm{bc}} \\
(26.50)\end{array}$ & $\begin{array}{l}9.61^{\mathrm{b}} \\
(92.0)\end{array}$ & $\begin{array}{c}6.72^{\mathrm{b}} \\
(45.0)\end{array}$ & $\begin{array}{c}6.64^{\mathrm{b}} \\
(43.80)\end{array}$ & $\begin{array}{l}2.56^{\mathrm{bc}} \\
(6.10)\end{array}$ & $\begin{array}{l}2.52^{\mathrm{c}} \\
(6.00)\end{array}$ & $\begin{array}{c}5.95^{\mathrm{b}} \\
(34.9)\end{array}$ & $\begin{array}{l}2.69^{b} \\
(6.75)\end{array}$ & $\begin{array}{c}7.05^{\mathrm{b}} \\
(49.25)\end{array}$ & 26.55 & 41.74 & 45.75 \\
\hline $\mathrm{T}_{6}$ & $\begin{array}{c}9.17^{\mathrm{bc}} \\
(84.00)\end{array}$ & $\begin{array}{c}5.08^{\mathrm{bc}} \\
(26.00)\end{array}$ & $\begin{array}{l}8.44^{\mathrm{c}} \\
(71.0)\end{array}$ & $\begin{array}{l}5.77^{\text {cd }} \\
(33.0)\end{array}$ & $\begin{array}{c}6.37^{\mathrm{bc}} \\
(40.32)\end{array}$ & $\begin{array}{c}2.52^{\mathrm{c}} \\
(5.98)\end{array}$ & $\begin{array}{l}2.95^{\mathrm{c}} \\
(8.25)\end{array}$ & $\begin{array}{l}5.24^{\mathrm{c}} \\
(26.9)\end{array}$ & $\begin{array}{l}2.33^{\text {cd }} \\
(4.95)\end{array}$ & $\begin{array}{c}6.19^{\mathrm{cd}} \\
(38.25)\end{array}$ & 36.59 & 59.80 & 53.42 \\
\hline $\mathrm{T}_{7}$ & $\begin{array}{c}9.17^{\mathrm{bc}} \\
(84.00)\end{array}$ & $\begin{array}{c}4.57^{\mathrm{c}} \\
(21.00)\end{array}$ & $\begin{array}{c}2.04^{\mathrm{e}} \\
(4.25)\end{array}$ & $\begin{array}{c}4.42^{\mathrm{ef}} \\
(19.50)\end{array}$ & $\begin{array}{l}2.54^{\mathrm{e}} \\
(6.12)\end{array}$ & $\begin{array}{l}2.28^{\mathrm{bc}} \\
(4.83)\end{array}$ & $\begin{array}{l}0.71^{\mathrm{d}} \\
(0.00)\end{array}$ & $\begin{array}{l}1.40^{\mathrm{e}} \\
(1.62)\end{array}$ & $\begin{array}{l}1.84^{\mathrm{ef}} \\
(2.93)\end{array}$ & $\begin{array}{c}0.71^{\mathrm{g}} \\
(0.00)\end{array}$ & 92.96 & 59.58 & 100.0 \\
\hline $\mathrm{T}_{8}$ & $\begin{array}{c}3.53^{\mathrm{e}} \\
(12.25)\end{array}$ & $\begin{array}{c}4.15^{\mathrm{c}} \\
(17.00)\end{array}$ & $\begin{array}{l}2.04^{\mathrm{e}} \\
(3.75)\end{array}$ & $\begin{array}{c}3.80^{\mathrm{f}} \\
(14.0)\end{array}$ & $\begin{array}{c}2.50^{\mathrm{e}} \\
(5.88)\end{array}$ & $\begin{array}{c}2.09^{\mathrm{c}} \\
(3.91)\end{array}$ & $\begin{array}{c}0.71^{\mathrm{d}} \\
(0.00)\end{array}$ & $\begin{array}{l}1.38^{\mathrm{e}} \\
(1.43)\end{array}$ & $\begin{array}{c}1.61^{\mathrm{f}} \\
(2.10)\end{array}$ & $\begin{array}{c}0.71^{\mathrm{g}} \\
(0.00)\end{array}$ & 93.21 & 68.95 & 100.0 \\
\hline $\mathrm{T}_{9}$ & $\begin{array}{c}0.71^{\mathrm{f}} \\
(0.00)\end{array}$ & $\begin{array}{c}4.14^{\mathrm{c}} \\
(16.75)\end{array}$ & $\begin{array}{c}0.71^{\mathrm{f}} \\
(0.00)\end{array}$ & $\begin{array}{c}3.77^{\mathrm{f}} \\
(13.75)\end{array}$ & $\begin{array}{c}0.71^{\mathrm{f}} \\
(0.00)\end{array}$ & $\begin{array}{c}2.08^{\mathrm{c}} \\
(3.85)\end{array}$ & $\begin{array}{c}0.71^{\mathrm{d}} \\
(0.00)\end{array}$ & $\begin{array}{c}0.71^{\mathrm{f}} \\
(0.00)\end{array}$ & $\begin{array}{c}1.60^{\mathrm{f}} \\
(2.06)\end{array}$ & $\begin{array}{c}0.71^{g} \\
(0.00)\end{array}$ & 100.0 & 73.45 & 100.0 \\
\hline $\mathrm{T}_{10}$ & $\begin{array}{c}11.54^{\mathrm{a}} \\
(133.2)\end{array}$ & $\begin{array}{c}7.28^{\mathrm{a}} \\
(52.75)\end{array}$ & $\begin{array}{l}10.7^{\mathrm{a}} \\
(115)\end{array}$ & $\begin{array}{c}8.27^{\mathrm{a}} \\
(68.0)\end{array}$ & $\begin{array}{c}8.01^{\mathrm{a}} \\
(63.96)\end{array}$ & $\begin{array}{c}3.55^{\mathrm{a}} \\
(12.1)\end{array}$ & $\begin{array}{c}5.74^{\mathrm{a}} \\
(32.50)\end{array}$ & $\begin{array}{l}6.64^{\mathrm{a}} \\
(43.7)\end{array}$ & $\begin{array}{c}3.27^{\mathrm{a}} \\
(10.2)\end{array}$ & $\begin{array}{c}8.39^{\mathrm{a}} \\
(70.25)\end{array}$ & 0.00 & 0.00 & 0.00 \\
\hline $\operatorname{SEm} \pm$ & 0.34 & 0.37 & 0.26 & 0.25 & 0.24 & 0.17 & 0.17 & 0.15 & 0.09 & 0.24 & - & - & - \\
\hline $\begin{array}{l}\mathrm{CD} \\
(p=0.05)\end{array}$ & 1.0 & 1.07 & 0.76 & 0.73 & 0.68 & 0.50 & 0.51 & 0.45 & 0.27 & 0.70 & - & - & - \\
\hline
\end{tabular}

$\mathrm{T}_{1}$ : Pendimethalin@ $750 \mathrm{~g} \mathrm{ha}^{-1} \mathrm{PE} ; \mathrm{T}_{2}$ : Pendimethalin@ $950 \mathrm{~g} \mathrm{ha}^{-1} \mathrm{PE}$ fbHW at $30 \mathrm{DAS} ; \mathrm{T}_{3}$ : Imazethapyr @ $75 \mathrm{~g} \mathrm{ha}^{-1} \mathrm{PE}$; $\mathrm{T}_{4}$ : Imazethapyr@ $75 \mathrm{~g} \mathrm{ha}^{-1} \mathrm{PE}$ fbHW at $30 \mathrm{DAS}$; $\mathrm{T}_{5}$ : Propaquizafop @ $75 \mathrm{~g} \mathrm{ha}^{-1}$ POE; $\mathrm{T}$ : Propaquizafop @ $75 \mathrm{~g} \mathrm{ha}^{-1}$ POE fbHW at 30 DAS; $\mathrm{T}_{7}$ : Pendimethalin+imazethapyr (pre-mixed) @ $800 \mathrm{~g} \mathrm{ha}^{-1} \mathrm{PE} ; \mathrm{T}_{8}$ : Pendimethalin+imazethapyr (pre-mixed) (a) $800 \mathrm{~g} \mathrm{ha}^{-1} \mathrm{PE}$ fbHW at $30 \mathrm{DAS} ; \mathrm{T}_{9}$ : IC fbHW at 20 and $40 \mathrm{DAS} ; \mathrm{T}_{10}$ : Weedy check

${ }^{*}$ Figures in the parenthesis are original values. All Figures are subjected to transformed values to square root $(\sqrt{ } \mathrm{x}+0.5)$. Figures indicating common letters in the column do not differ significantly from each other at $5 \%$ level of significance according to Duncan New Multiple Range Test

interculturing followed by hand weeding at 20 and 40 days after sowing. The seed and stover yield were also significantly higher under interculturing followed by hand weeding carried out at 20 and 40 days after sowingthan rest of the treatments but wasat par with pendimethalin+imazethapyr (pre-mixed) (a) $800 \mathrm{~g} \mathrm{ha}^{-1}$ pre-emergencefollowed byhand weedingat 30 days after sowing, whereas weedy check recorded the lowest yield attributes, seed and stover yield of cluster bean due to higher weed density (Table 2). Removal of weeds at early stage in the season reduced crop weed competition at the lowest possible limit and provided almost weed free environment.
Due to controlling weeds, higher growth and yield parameters of cluster bean were probable reasons for higher seed yield in interculturing+hand weeding treatment. These results are in accordance with the findings of Yadav et al. (2011); Soltani et al. (2012); Bhadoria and Jain (2005). The pre-emergence application of pendimethalin+imazethapyr (pre-mixed) gave excellent control and suppression of weeds in the season was probably effective against secondary weed emergence at 10 to 15 days after sowing stage onwards due to persistent of imazethapyr for long period. The weed management schedule having low weed biomass and higher weed control efficiency 
Table 2: Weed index, plant height, crop dry matter accumulation, number of branches, yield attributes, yield and economics of cluster bean as influenced by different weed management practices economics

\begin{tabular}{|c|c|c|c|c|c|c|c|c|c|c|c|c|}
\hline \multirow{2}{*}{$\begin{array}{l}\text { Treat- } \\
\text { ments }\end{array}$} & \multirow{2}{*}{$\begin{array}{l}\text { Weed } \\
\text { index } \\
(\%)\end{array}$} & \multicolumn{3}{|c|}{ Plant height $(\mathrm{cm})$} & \multirow{2}{*}{$\begin{array}{c}\text { Crop dry } \\
\text { matter } \\
\text { accumulation } \\
\text { at } 50 \text { DAYS } \\
\text { after sowing }(\mathrm{g})\end{array}$} & \multirow{2}{*}{$\begin{array}{l}\text { Num- } \\
\text { ber of } \\
\text { branches } \\
\text { plant }^{-1}\end{array}$} & \multirow{2}{*}{$\begin{array}{l}\text { Number } \\
\text { of pods } \\
\text { plant }^{-1}\end{array}$} & \multirow{2}{*}{$\begin{array}{c}\text { Test } \\
\text { weight } \\
(\mathrm{g})\end{array}$} & \multirow{2}{*}{$\begin{array}{c}\text { Seed } \\
\text { yield } \\
\left(\mathrm{kg} \mathrm{ha}^{-1}\right)\end{array}$} & \multirow{2}{*}{$\begin{array}{c}\text { Stover } \\
\text { yield } \\
\left(\mathrm{kg} \mathrm{ha}^{-1}\right)\end{array}$} & \multirow{2}{*}{$\begin{array}{c}\text { Net } \\
\text { return } \\
(₹ \text { ha) }\end{array}$} & \multirow{2}{*}{$\begin{array}{l}\mathrm{B}: \mathrm{C} \\
\text { ratio }\end{array}$} \\
\hline & & $\begin{array}{c}30 \\
\text { DAS }\end{array}$ & $\begin{array}{c}60 \\
\text { DAS }\end{array}$ & $\begin{array}{c}\text { At } \\
\text { Harvest }\end{array}$ & & & & & & & & \\
\hline $\mathrm{T}_{1}$ & 24.81 & $42.88^{\mathrm{bcd}}$ & $105.13^{\mathrm{abc}}$ & $149.48^{\mathrm{abc}}$ & $78.50^{\mathrm{abc}}$ & $11.15^{\mathrm{ab}}$ & $159.65^{\mathrm{ab}}$ & $32.25^{\mathrm{ab}}$ & $125^{6 b}$ & $45^{\circ}$ & 41956 & 2.19 \\
\hline $\mathrm{T}_{2}$ & .81 & $39.30^{\text {cde }}$ & $101.0^{\mathrm{abcd}}$ & $150.05^{\mathrm{abc}}$ & $80.63^{\mathrm{ab}}$ & $5^{\mathrm{ab}}$ & $166.23^{\mathrm{ab}}$ & $32.75^{\mathrm{ab}}$ & $1301^{b}$ & $4610^{\mathrm{a}}$ & 42937 & 2.13 \\
\hline $\mathrm{T}_{3}$ & 27.63 & $34.55^{\mathrm{e}}$ & $96.53^{\mathrm{bcd}}$ & $143.65^{\mathrm{cd}}$ & $76.13^{\mathrm{abc}}$ & $10.95^{\mathrm{ab}}$ & $149.05^{\mathrm{bc}}$ & $32.00^{\mathrm{ab}}$ & $1194^{b}$ & $4394^{\mathrm{ab}}$ & 38639 & 1.98 \\
\hline $\mathrm{T}_{4}$ & 24.82 & $31.63^{\mathrm{e}}$ & $93.10^{\mathrm{cd}}$ & $147.03^{\mathrm{bcd}}$ & $78.00^{\mathrm{abc}}$ & $11.00^{\mathrm{ab}}$ & $156.10^{\mathrm{ab}}$ & $32.00^{\mathrm{ab}}$ & $1249^{b}$ & $4451^{\mathrm{ab}}$ & 40141 & 1.96 \\
\hline $\mathrm{T}_{5}$ & 47.92 & $50.10^{\mathrm{ab}}$ & $95.40^{\text {bcd }}$ & $150.40^{\mathrm{abc}}$ & $65.53^{\mathrm{bcd}}$ & $9.90^{\mathrm{b}}$ & $116.30^{\mathrm{d}}$ & $31.00^{\mathrm{b}}$ & $871^{\mathrm{cd}}$ & $3772^{b}$ & 23669 & 1.23 \\
\hline $\mathrm{T}_{6}$ & 35.12 & $44.08^{\mathrm{bc}}$ & $97.88^{\mathrm{bcd}}$ & $151.08^{\mathrm{abc}}$ & $63.53^{\mathrm{cd}}$ & $11.15^{\mathrm{ab}}$ & $125.45^{\mathrm{cd}}$ & $31.75^{\mathrm{b}}$ & $1096^{\mathrm{bc}}$ & $4267^{\mathrm{ab}}$ & 33258 & 1.64 \\
\hline $\mathrm{T}_{7}$ & 22.23 & $35.95^{\mathrm{de}}$ & $105.98^{\mathrm{ab}}$ & $152.68^{\mathrm{abc}}$ & $82.60^{\mathrm{a}}$ & $11.40^{\mathrm{ab}}$ & $173.30^{\mathrm{ab}}$ & $33.00^{\mathrm{ab}}$ & $1304^{b}$ & $4775^{\mathrm{a}}$ & 43601 & 2.20 \\
\hline $\mathrm{T}_{8}$ & 17.88 & $37.30^{\text {cde }}$ & $108.05^{\mathrm{ab}}$ & $158.08^{\mathrm{ab}}$ & $84.90^{\mathrm{a}}$ & $11.60^{\mathrm{ab}}$ & $177.73^{a}$ & $33.25^{\mathrm{ab}}$ & $1375^{\mathrm{ab}}$ & $4881^{\mathrm{a}}$ & 45872 & 2.20 \\
\hline $\mathrm{T}_{9}$ & 1.94 & $44.15^{\mathrm{bc}}$ & $113.38^{\mathrm{a}}$ & $162.05^{\mathrm{a}}$ & $87.48^{\mathrm{a}}$ & $12.15^{\mathrm{a}}$ & $182.08^{\mathrm{a}}$ & $34.25^{\mathrm{a}}$ & $1655^{\mathrm{a}}$ & $4936^{a}$ & 59404 & 2.97 \\
\hline $\mathrm{T}_{10}$ & 51.76 & $53.65^{\mathrm{a}}$ & $90.08^{\mathrm{d}}$ & $136.50^{\mathrm{d}}$ & $59.8^{d}$ & $7.85 \mathrm{c}$ & $106.90^{\mathrm{d}}$ & $28.75^{\mathrm{d}}$ & $800^{\mathrm{d}}$ & $2477^{\mathrm{c}}$ & 21305 & 1.24 \\
\hline $\mathrm{SEm} \pm$ & - & 2.41 & 3.86 & 3.82 & 4.87 & 0.61 & 8.17 & 0.74 & 88.97 & 245.72 & - & - \\
\hline $\begin{array}{l}\text { CD } \\
(p=0.05)\end{array}$ & - & 6.99 & 11.19 & 11.09 & 14.13 & 1.76 & 23.71 & 2.15 & 258.20 & 713.07 & - & - \\
\hline
\end{tabular}

$\mathrm{T}_{1}$ : Pendimethalin@ $750 \mathrm{~g} \mathrm{ha}^{-1} \mathrm{PE} ; \mathrm{T}_{2}$ : Pendimethalin@ $750 \mathrm{~g} \mathrm{ha}^{-1}$ PEfbHW at $30 \mathrm{DAS} ; \mathrm{T}_{3}$ : Imazethapyr @ $75 \mathrm{~g} \mathrm{ha}{ }^{-1} \mathrm{PE} ; \mathrm{T}_{4}$ : Imazethapyr@ $75 \mathrm{~g} \mathrm{ha}^{-1}$ PE fbHW at $30 \mathrm{DAS}$; $\mathrm{T}_{5}$ : Propaquizafop@ $75 \mathrm{~g} \mathrm{ha}^{-1} \mathrm{POE}$; $\mathrm{T}$ : Propaquizafop @ $75 \mathrm{~g} \mathrm{ha}^{-1} \mathrm{POE}$ fbHW at 30 DASO; $\mathrm{T}_{7}$ : Pendimethalin+imazethapyr (pre-mixed) @ $800 \mathrm{~g} \mathrm{ha}^{-1} \mathrm{PE} ; \mathrm{T}_{8}$ : Pendimethalin+imazethapyr (pre-mixed) @ $800 \mathrm{~g} \mathrm{ha}^{-1} \mathrm{PE}$ fbHW at $30 \mathrm{DAS} ; \mathrm{T}_{9}$ : IC fbHW at 20 and $40 \mathrm{DAS} ; \mathrm{T}_{10}$ : Weedy check

"Figures indicating common letters in the column do not differ significantly from each other at $5 \%$ level of significance according to Duncan New Multiple Range Test

would provide favorable situation for maximization of cluster bean yield potential.Results were conformity with the finding of Yadav et al. (2011); Jha and Soni (2013).

\subsection{Economic implication}

Net monetary returns and $\mathrm{B}: \mathrm{C}$ ratio was higher under the interculturing followed byhand weeding at 20 and 40 days after sowing than other weed management practices. Patel et al. (2005) also reported maximum additional profit over control in twice interculturing followed by hand weeding carried out at 30 and 45 days after sowing. Among herbicide application ofpendimethalin+imazethapyr (pre-mixed) @ $800 \mathrm{~g} \mathrm{ha}^{-1}$ pre-emergence followed by hand weeding at 30 days after sowing recorded higher net return and B:C ratio. Jha and Soni (2013) reported same trend in case of herbicide combination.

\section{Conclusion}

For effective control of weeds and for securing higher seed yield of cluster bean as well as economical returns, interculturing followed by hand weeding at 20 and 40 days after sowingand in Integrated weed management approach application of pendimethalin+imazethapyr (pre-mixed) @ 800 $\mathrm{g} \mathrm{ha}^{-1}$ as pre-emergence followed by hand weeding at 30 days after sowingis better.

\section{References}

Anonymous, 2011. Crops statistics of Gujarat. Department of Agriculture, Government of Gujarat. http://agri.gujarat. gov.in.

Anonymous, 2012. Area, Production and Productivity of different crops in India. Directorate of Economics and Statistics, Department of Agriculture and Cooperation. http://agricoop.nic.in/Agristatistics.

Arora, S.S., 1979. Guar-a versatile crop. Science Reporter 16(5), 337-340.

Bhadoria, R.B.S., Jain, P.C., 2005. Crop-weed competition studies on cluster bean under rainfed condition. Forage Research 31(2), 97-98.

Gandhi, T., Shakhela, R.R., 2005. Effect of P and S and their interaction on yield, protein content and gum content of cluster bean [Cyamopsis tetragonoloba (L.) taub.] crop. GAU Research journal 30(1\&2), 40-42.

Jain, V., Yadav, B.D., Sharma, B.D., Taneja, K.D., 1987. Effect 
of dates, row spacing and varieties on yield and quality of cluster bean (Cyamopsis tetragonoloba L.). Indian Journal of Agronomy 32(4), 378-382.

Jha, A.K., Soni, M., 2013. Weed Management by sowing methods and Herbicides in soybean. Indian Journal of Weed Science 45(4), 250-252.

Patel, B.D., Patel, R.B., Meisuriya, M.I., Patel, M.V., 2005. Integrated weed management in clusterbean. In: National Biennial Conference, Indian Society of Weed Society, Panjab Agricultural University, 154-156.

Punia, S.S., Singh, S., Yadav, D., 2011. Bioefficacy of Imzethapyar and Chlorimuron-ethyl in Cluster bean and their residual effect on succeeding rabi crops. Indian Journal of Weed Science 43(1\&2), 48-53.

Soltani, N., Robert, E., Peter, H., 2012. Weed control in dry bean with pendimethalin plus reduced rates of imazethapyr. International Research Journal of Agricultural Science and Soil Science 2(8), 312-317.

Yadav, S.L., Kaushik, M.K., Mundra, S.L., 2011. Effect of weed control practices on weed dry weight, nutrient uptake and yield of Cluster bean [Cyamopsis tetragonoloba (L.) taub.] under rainfed condition. Indian Journal of Weed Science 43(1\&2), 81-83. 